\title{
Calculation of the Boundary Dimensions of Functionally Active Nanoparticles
}

\author{
Zulayho A. Smanova, National University of Uzbekistan, Uzbekistan \\ Tokhir Kh. Rakhimov, National University of Uzbekistan, Uzbekistan \\ (iD https://orcid.org/0000-0002-5755-5918 \\ Muxtarjan Mukhamediev, National University of Uzbekistan, Uzbekistan \\ Dilfuza Gafurova, National University of Uzbekistan, Uzbekistan \\ Dilbar Shaxidova, National University of Uzbekistan, Uzbekistan
}

\begin{abstract}
Size is a key characteristic of nanoparticles that determines whether the objects belong to this category. Currently, there are not enough experiments on materials of the same chemical composition but of different dispersion in particle size, with equal size of the particles or grains of each sample of material investigated. In the present article, the authors show that the effect of the dispersion of the particle size determines whether the size dependence of a specific property can be calculated alternatively to the direct measurements. By finding the correlations between nano-properties and content of nanoparticles' fractions of different sizes, the boundary conditions can be calculated.
\end{abstract}

\section{KEYWORDS}

Air Purification, Carbon Monoxide, Life Support, Mathematical Modeling, Nanocatalysts, Nanoparticles, Palladium

\section{INTRODUCTION}

It became quite obvious that not only the composition, but also the size of nanoparticles can have a key effect on their properties (Gajewicz et al, 2015, Rasulev et al, 2012), in particular, on the catalytic activity. Catalytic processes depend on the coordination of atoms. The atoms located on the faces have a higher coordination than the atoms on the vertices and edges (Ni \& Wang 2015). If atoms in low coordination are the most catalytically active, the catalytic activity increases with decreasing particle size. In another case, if the atoms located on the faces are catalytically active, the rate of the catalyzed reaction will be increased by larger particles.

This approach provides an understandable qualitative explanation, but does not allow quantitative determination of the particle size boundaries in which the catalytic effect persists (Wang \& Lu 2020). In the available open-access literature, it was not possible to find methods for calculating the position of these boundaries, although approaches to quantitatively predicting the size of particles during their formation are being successfully developed (Handwerk et al., 2020). 
Classic monograph on Nanotechnology (Gusev \& Rempel 2004) emphasizes: "The main scope of investigations of the nanocrystalline state is answering the following questions: "Is there a sharp, distinctive boundary between the bulk and the nanocrystalline states? Is there some critical grain (particle) size at which the characteristic properties of nanocrystals become observable, and above which the material behaves as a bulk one?" In addition - not only below, but also above. This main scope may be decided by using the mathematic modelling approaches.

This investigation is an attempt to find in first approximation approach the solution to this important question. The best example for application of this approach can be the case when nanoproperty is observed on medium size particles. One of such system is present in nano-sized catalysts. It is known that if the atoms located on the faces are catalytically active, the rate of the catalyzed reaction will be increased by larger surfaces of particles (Goodman 1994).

\section{OBJECTS OF EXPERIMENT}

As the quantitative statistical characteristics reflecting the average relative values of the active surface of nanomaterials are prompted to introduce the rms diameter and polydispersity index of nanoparticles (Rakhimov 2014). The question is: from which size dimension of particles some special properties getting acquired, namely are there nano-structure properties appearing, and at what size dimensions are they appearing? What can be considered as a nanoparticle with all their acquired features?

Special drawing catalysts comprising platinum metals, palladium and platinum, as active ingredients, were investigated, Table 1. Fiber polymers - polypropylene fiber, polyacrylonitrile, carbon fibers, low modulus carbon, fibrous materials obtained by carbonization and subsequent activation of mats from "Mtilon-M", copolymers of polyacrylonitrile and hydrocellulose, were used as bearers.

The possibility of exhaustive removal of toxic components, mainly $\mathrm{CO}$, from air by passive systems has been achieved with advent of nanocatalysts (Rakhimov et al., 2014). A decisive influence of substrates nature on the activity and stability and the process character was determined (Rakhimov et al., 2014; Nurmukhammadov et al., 2014; Smanova et al., 2011; Madusmanova et al., 2020). At the same time, it is obvious that the state and also physical and chemical characteristics of active component will exert not smaller influence on properties of the catalytic system in whole.

Activity of catalysts was determined by decreasing the $\mathrm{CO}$ content in gas-air mixture at room temeprature. The rms diameter and coefficient of polydispersity of nanoparticles (Rakhimov 2014) were measured by transmission electron microscopy, Table 1 .

\section{RESULTS AND DISCUSSION}

\section{Catalytic Activity as A Special Nanoparticles Property}

For investigation of the influence of qualitative changing of nano-composites properties the most evident observations of peculiar properties related specifically to nano-particles can be used. As such peculiar properties can be attributed to effect shown by drawing palladium nano-catalysts at low-temperature oxidation of $\mathrm{CO}$ by oxygen. The exhaustive removal of $\mathrm{CO}$ by oxygen's catalytic oxidation in air at room temperature in real experiments do not usually traced neither for too small (solutions, solid solutions) nor for too large (large particles) objects of similar chemical compositions (Rakhimov et al., 2014).

\section{Are There Any Exclusive Properties of Nanoparticles?}

Before construction of the model, it is necessary to state the following: there are present or not present some qualitative, spasmodic changes in properties inherited to substances characterized specifically by nano-sized dimensions. If such changes are absent, then only quantitative inter-relationship of 
Table 1. Activity of palladium-containing nano-catalysts (4 mass.\%) with different average squared diameters of particles in following regime.*

\begin{tabular}{|c|c|c|c|}
\hline $\begin{array}{l}\text { Average squared } \\
\text { diameter, } \mathbf{n m}\end{array}$ & $\begin{array}{r}\text { Specific } \\
\text { activity*10 } \\
\text { mole/l*s*g }\end{array}$ & $\begin{array}{l}\text { Increasing of total } \\
\text { surface in times }\end{array}$ & $\begin{array}{l}\text { Increasing of specific activity } \\
\text { in calculation on the unit of area of } \\
\text { surface in times }\end{array}$ \\
\hline \multicolumn{4}{|c|}{ Bearer - activated carbon fibrous materials from Mtilon-M, active surface area $2700 \mathrm{~m}^{2} / \mathrm{g}$} \\
\hline 2000 & 0.2 & 1 & 1 \\
\hline 1500 & 1.4 & 1.3 & 5 \\
\hline 1200 & 1.12 & 1.7 & 3 \\
\hline 1000 & 15.6 & 2 & 39 \\
\hline 800 & 22.1 & 2.5 & 44 \\
\hline 720 & 21.1 & 2.8 & 38 \\
\hline 601 & 24 & 3.3 & 36 \\
\hline 511 & 27.5 & 3.9 & 35 \\
\hline 380 & 15.1 & 5.3 & 14 \\
\hline 222 & 6.4 & 9 & 4 \\
\hline 104 & 2.9 & 19.2 & 0.8 \\
\hline 62 & 0.8 & 32.3 & 0.1 \\
\hline 47 & 0.2 & 42.6 & 0 \\
\hline 24 & 0 & 83.3 & 0 \\
\hline 12 & 0 & 166.7 & 0 \\
\hline \multicolumn{4}{|c|}{ Bearer -carbon fibrous materials from hydrocellulose, active surface area $300 \mathrm{~m}^{2} / \mathrm{g}$} \\
\hline 200 & 0 & & \\
\hline 120 & 0.11 & 1 & 1 \\
\hline 111 & 0.09 & 1.1 & 0.8 \\
\hline 98 & 0.84 & 1.2 & 6.2 \\
\hline 85 & 1.4 & 1.4 & 9 \\
\hline 77 & 1.12 & 1.6 & 6.5 \\
\hline 64 & 1.16 & 1.9 & 5.6 \\
\hline 54 & 1.21 & 2.2 & 5 \\
\hline 44 & 1.21 & 2.7 & 4 \\
\hline 36 & 0.41 & 3.3 & 1.1 \\
\hline 28 & 0.27 & 4.3 & 0.6 \\
\hline 19 & 0.12 & 6.3 & 0.2 \\
\hline 10 & 0 & 12 & 0 \\
\hline Less than 10 & 0 & 24 & 0 \\
\hline
\end{tabular}

${ }^{*}$ Bold italics are attributed to data based on which the calculations were carried out.

smooth changes in properties with parameters connected to nano-size dimensions of particles must be presented. 
Figure 1. Microphotography of activated carbon fiber obtained by carbonization of Mtilon-M with drawing palladium.

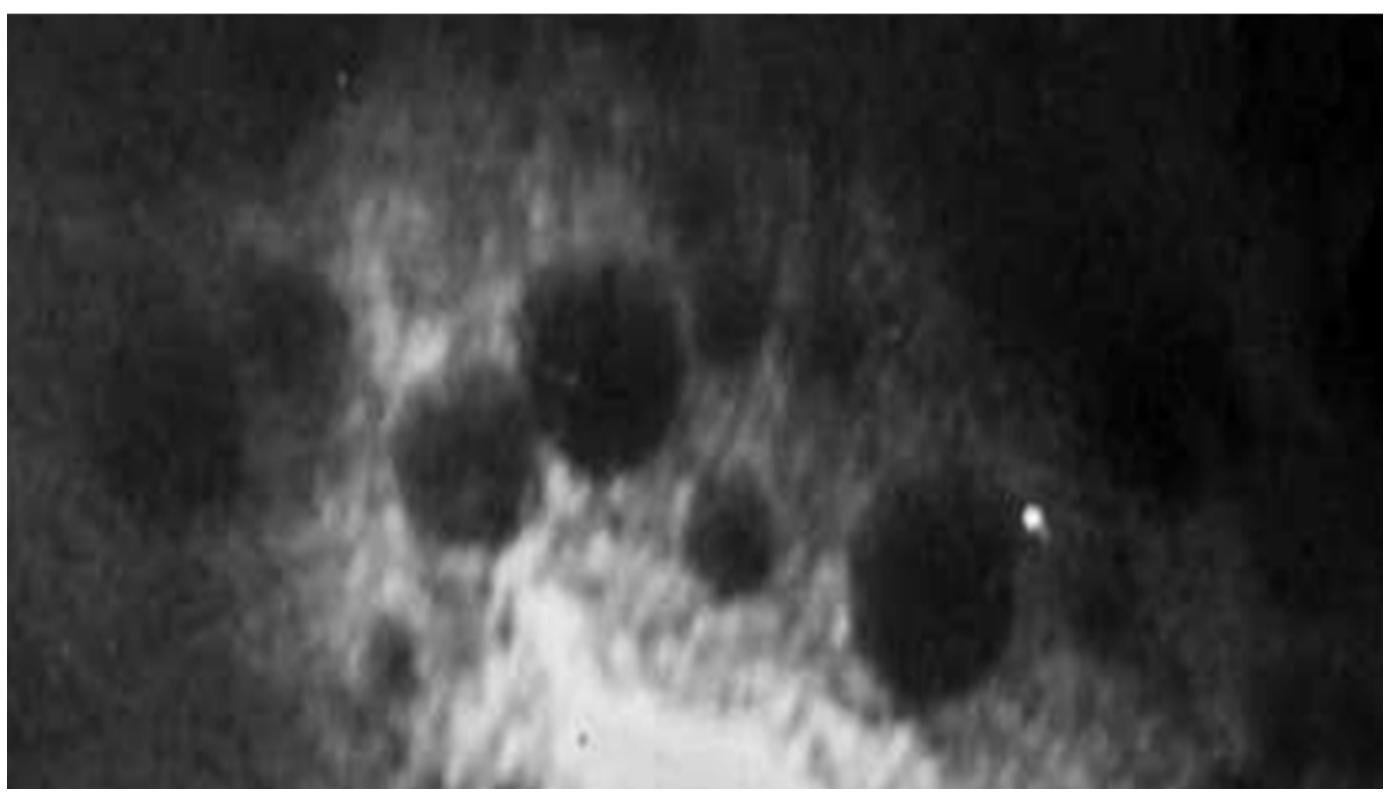

Let us assume that, with the manifestation of high catalytic activity of composites, there are no specific effects associated with nanoscale size. In this case, the rate of catalytic heterogeneous reactions will correlate with the value of the total surface area of all particles available to the reactants. Therefore, the catalytic activity should gradually increase with increasing of the total surface area. Even if the correlation is not strictly linear, there should be no abrupt changes in the relationship curve of the catalytic activity with the size change of catalyst particles. Since the volume is proportional to the cube of the radius of sphere, and the surface area is proportional to the square of the diameter of ball, the total surface area will increase by the same amount as the radius decreases.

Comparison of catalytic activity of samples with palladium on the fiber obtained by carbonization and following activation of Mtilon-M is showed that there is a sharp increasing of activity for nanoparticles with size dimensions of 300-1000 nm (Table 1). The same qualitative changing of catalytic activity was observed for samples on the base of carbonized hydrocellulose with dimensions of particle of 40-100 nm (area of active surface $-300 \mathrm{~m}^{2} / \mathrm{g}$ ). Since larger surface sized nanoparticles are active, the most catalytically active in this process are highly coordinated atoms located on the faces, rather than at the sites and edges of the crystal lattice (Karpiński, Z. 1990).

These data observed are in benefit of hypothesis about presence of clearly expressed effect of increasing the catalytic activity when increasing the content of nanoparticles of definite radiuses. Apparently due to the marked sizes of particles with acceptable deviations from average values responsible for catalytic activity, the active particles are nano-structures with narrow limits of particles dimensions.

Based on obtained data it is shown that effect of spasmodic increased activity for composites with definite average dimensions of particles can't be explained by increasing of the total surface of contact of particles with substrate. Thus, it is determined that there are effects caused by nano-sized dimensions of composite's particles.

\section{Bordering Conditions of Exclusive Properties of Nanocatalysts}

In this regard, the logical the second question is - From what size dimension of particles the nanoproperties are appearing and at what minimal/maximal size dimension the properties are dissapearing? 
In other words - How to determine those limits at which particles can be called nanoparticles and composites - nanocomposites? It is clear that for each system these values will be different.

Estimation of limits of minimal and maximal size dimensions of nanoparticles outside of which "nano properties" are disappearing is impossible without expensive mathematical approaches. For statement of above-mentioned task, the evident model is proposed which allowed to simplify the understanding of chemical problems by mathematicians. This model conditionally was named the "model of balls coloring". Below is the description of this model.

\section{MODEL OF BALLS COLORING}

\section{Description of Task}

\section{Hypothetical case}

Let's assume that it is necessary to paint by different colors the surface of balls which in reality are ellipsoids stretching in one direction, but in first approach we shall suppose them as balls. Color in this case will be corresponded to definite experimental sample possessed by measured macro-properties - in our case it is correspondent nano-catalysts and their catalytic activity. Balls are in containers. In each container there are enough number of balls - about 10 billion and by this reason the statistical regularities can be used. The common volume (or mass) of all balls for all containers is similar (naturally, the poured volume of balls will be different, but this fact isn't important). Balls from each container must be painted by the same color, but from different containers - by different colors.

However, not all balls must be colored. It is decided to not to paint too small and too large balls. There are known different and limited amounts of dyes for each color. Expenditure of dye for the unit of surface of balls is the same. It is obvious that these amount from the point of view of chemist will correspond to observed and measurable macro-property - in our case to catalytic activity determined as a rate of oxidation reaction of $\mathrm{CO}$ in the presence of given concrete sample of catalyst.

\section{Required To Determine}

It is necessary to select such values of minimal and maximal radiuses of balls at which dye will be spent optimally.

\section{Measured and Available Data}

Before carrying out of calculations, from each container of not less than 40 balls were selected randomly and their radiuses are measured. In result for each container two characteristics were obtained:

average squared radius of ball which was calculated on the base of experimental results by formula:

$$
\bar{R}=\sqrt{\frac{\sum_{i=1}^{n} R_{i}^{2}}{n}}
$$

and the coefficient of polydispersity L - which is indifferent value (expressed in \%) and characterized by irregularity of nanoparticles size dimensions. This parameter was calculated on the base of experimental data by formula: 
Table 2. Listed data that used to build model of balls coloring. Data compiled on the basis of experimental data of measured catalytic activity of activated carbon fibers based on Mtilon-M with drawing palladium nanoparticles

\begin{tabular}{|c|c|c|c|}
\hline Color of painted balls & Average squared radius, $\mathbf{m m}$ & $\mathrm{L}, \%$ & Volume, $\mathbf{m}^{3}$ \\
\hline Red & 125 & 144 & 1.12 \\
\hline Orange & 263 & 90 & 1.28 \\
\hline Yellow & 224 & 98 & 1.92 \\
\hline Green & 336 & 60 & 2.56 \\
\hline Blue & 301 & 71 & 3.28 \\
\hline Dark blue & 222 & 95 & 4.48 \\
\hline Violet & 336 & 57 & 4.8 \\
\hline Brown & 349 & 49 & 9.84 \\
\hline White & 268 & 38 & 22 \\
\hline Black & 6 & 81 & 0.08 \\
\hline
\end{tabular}

$L=\frac{\sum_{i=1}^{n}\left(\bar{R}-R_{i}\right)^{2}}{n \bar{R}^{2}}$

Quantity of dye (in given case it corresponded to rate of reaction in the presence of given sample) was known in advance (in given case this quantity was obtained in results of experiments on the base of measuring of catalytic activity that is by decreasing of $\mathrm{CO}$ concentration on the outlet).

As an example, data obtained on the base of real measurements by rate determination $(\mathrm{V})$ of catalytic reaction for activated carbon fibers based on Mtilon-M with drawing palladium nanoparticles are presented below (Table 2). To make it clear to the mathematical problem values "nm" were substituted for "mm" and V - for volume in $\mathrm{m}^{3}$.

Also, the function of balls distribution by their dimensions is necessary for carrying out of abovementioned calculation. We have supposed that this distribution is normal - Gaussian distribution.

\section{Principles of Calculation}

It is necessary to introduce values of minimal $\mathrm{m}$ and maximal $\mathrm{M}$ radiuses outside of which coloring do not occur. To find the necessary quantity of dye it is important to calculate the total surface area of colored balls.

For each container, the values of the total surface area of balls is calculated. For each i-ball:

$\mathrm{S}_{\mathrm{i}}=4 \pi \mathrm{R}_{\mathrm{i}}^{2}$

If the radiuses of balls are changed on small value $\mathrm{dR}$, then for determination of the total surface area of balls it is necessary to calculate some determined integral:

$S=\int_{m}^{M} 4 \pi f\left(R^{2}\right) d R$ 
Figure 2. Total surface of painted balls.

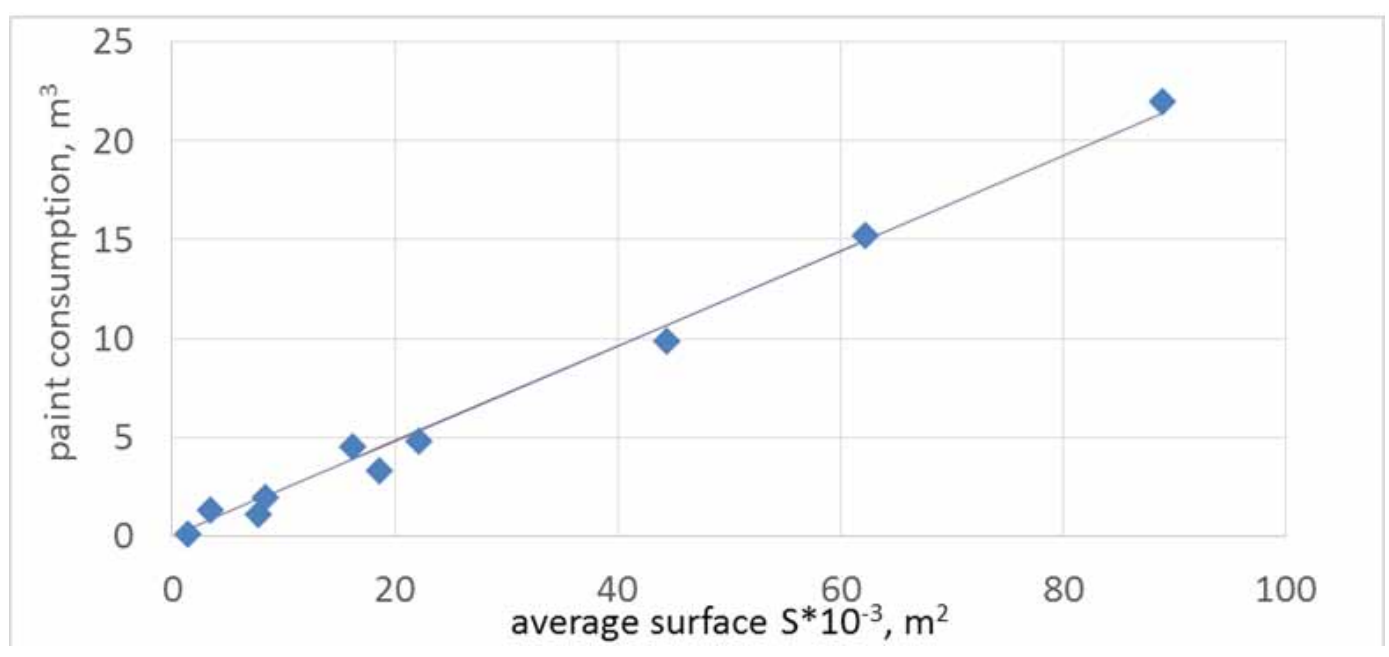

Function $f\left(R^{2}\right)$ is constructed analytically on the basis of data related to type of distribution of balls by size dimensions - Gaussian distribution; values of their average dimension and coefficient of polydispersity.

In result, we were able to obtain the analytical method of determination of the total surface area of all painted balls from each container depending on given values of "m" and "M" (Fig. 2).

Determination of average squared deviation (mean error). After calculation of analytically total surface of dying balls for each container, the dependence of calculated and factual amount of dye was constructed. Deviations were determined by method of differentiation (for example, by partial least squares method). By given values of " $\mathrm{m}$ " and "M" it is possible to find values of mean error.

Also, for better understanding it is possible to make the following conclusion: "the more balls in the container of average size dimension the more amount of dye is required". And this fact also is dependent on average dimension of balls, type of distribution and values of polydispersity - the more evenly sized balls are distributed around of average size value the more amount of dye will be needed and also dispersion of eliminated for dying balls will be narrow.

Then formula of deviation value (confidential interval) at fixed probability for given values " $m$ " and " $\mathrm{M}$ " is determined. This formula will be the final formula for our model.

Now there is a formula by which it is possible to find values of the confidential interval $\mathrm{X}$ or average squared deviations. By given values of " $\mathrm{m}$ " and " $\mathrm{M}$ " in this formula sought for values of interval boundaries of balls' sizes will be supposed those for which confidential interval is minimum, that there is a coincidence of calculated values with experimental data (Fig. 3).

These values can be obtained by the following method. At given step for " $\mathrm{m}$ " and "M" X can be calculated. Geometrically this will be the surface where " $\mathrm{m}$ " and "M" will be correspondently abscissa and ordinate, and X - applicate. The minimum value of this surface is determined and then values of " $m$ " and "M" can be calculated. For specific nanosystems (in our case - nano-catalysts), the values of " $\mathrm{m}$ " and "M" are allowed to give nanostructures the quantitative determination and to determine the real boundaries inside of which the special nano-properties are displayed.

The proposed model has broad prospects for adaptation to specific objects of investigation. In some cases, it may be necessary to take into account other factors, for example, the nonlinearity of the dependence of nanostructure properties vs surface area; the shape of nanoparticles can differ from spherical or ellipsoidal shape; structure and chemical properties can differ for particles of different sizes, as well as within the same particle (anisotropy). The value of the proposed approach lies in 
Figure 3. Find the value of minimal $m$ and maximal $M$ balls radiuses, corresponding to the minimum total error $\Omega$.

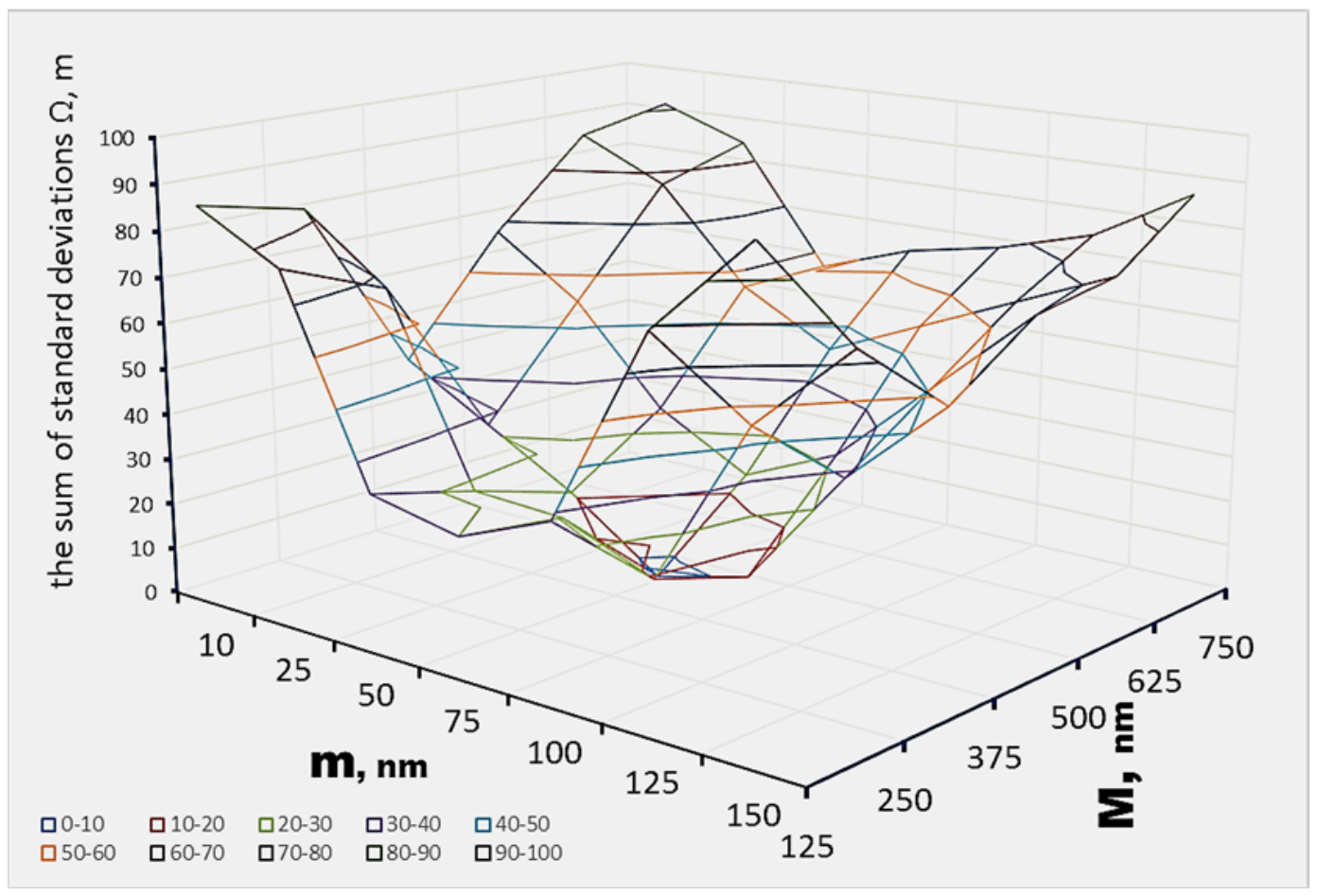

the fact that for the first time it opens up the possibility of a quantitative assessment of the position of the dimensional boundaries of functionally active nanoparticles.

\section{CONCLUSION}

In this paper the proofs in a simple way are discussed about qualitative differences of the properties of nanoparticles from sizes of objects, which are within the limits of nano-dimensions. On the example of high-effective polymeric nanocatalysts of low temperature oxidation of $\mathrm{CO}$ it was shown in such nano-composites the critical function is a content of the particles of an active component, with definite dimensions values which are inside of clearly designated limiting values that are different for different systems. The limits inside the borders are determining the qualitative peculiarity of nanostructure properties.

Mathematical model was proposed which allowed to determine limits of nanoparticles size dimensions within the boundaries at which they displayed special properties inherited to only nanostructures. The model was allowed to make the decisions related to finding the values of maximum and minimum radiuses of particles, in particular, for catalytically active nanocomposites. The model also can be expanded to explain other special properties inherited only to nano-systems. 


\section{REFERENCES}

Gajewicz, A., Cronin, M. T. D., Rasulev, B., Leszczynski, J., \& Puzyn, T. (2015). Novel approach for efficient predictions properties of large pool of nanomaterials based on limited set of species: Nano-read-across. Nanotechnology, 26, 015701.

Goodman, D. W. (1994). Catalysis: from single crystals to the "real world". Surface Sci. 837-848.doi. org/10.1016/0039-6028(94)90701-3

Gusev, A. I., \& Rempel, A. A. (2004). Nanocrystalline Materials. Cambridge Int. Sci. Publ.

Handwerk, D. R., Shipman, P. D., Whitehead, C. B., Özkar, S., \& Finke, R. G. (2020). Particle Size Distributions via Mechanism-Enabled Population Balance Modeling. The Journal of Physical Chemistry C, 124(8), 4852-4880. doi:10.1021/acs.jpcc.9b11239

Karpiński, Z. (1990). Catalysis by supported, unsupported, and electron-deficient palladium. Advances in Catalysis, 37, 45-100.

Madusmanova, N. K., Smanova, Z. A., \& Joryev, I. I. (2020). Properties of a new analytical reagent 2-hydroxy3-nitrosomethylurea. Journal of Analytical Chemistry, 75(1), 92-96. doi:10.31857/S0044450220010107

Ni, B., \& Wang, X. (2015). Face the edges: Catalytic active sites of nanomaterials. Advancement of Science, 2(7), 1500085. doi:10.1002/advs.201500085 PMID:27980960

Nurmukhammadov, J., Smanova, Z. A., Tojimukhamedov, H., \& Inatova, M. S. (2014). Synthesis and properties of a new analytical reagent, 2-hydroxy-3-nitrosonaphthalene-1-carbaldehyde. Russian Journal of Organic Chemistry, 50(6), 895-897.

Rakhimov, T. Kh. (2014). Quantitative Criteria For The Comparative Size Of The Nanoparticles. $8^{\text {th }}$ Int. Symp. "Molecular Order and Mobility in Polymer Systems". www.spsl.nsc.ru/FullText/konfe/obl2014.pdf

Rakhimov, T. Kh., \& Mukhamediev, M. G. (2014). Palladium containing composite nanosystems in lowtemperature co oxidation: decisive influence of the support to the reactions' mode. Composite Materials Uzbekistan Sci. and Tech. J., 2, 8-11.

Rakhimov, T. Kh., Mukhamediev, M. G., \& Khakimjanov, B. Sh. (2014). The Synthesis of Applied NanoCatalysts of Low-Temperature Carbon Monoxide Oxidation. 8th Int. Symp. "Molecular Order and Mobility in Polymer Systems”, 116. www.spsl.nsc.ru/FullText/konfe/obl2014.pdf

Rasulev, B., Watkins, M., Theodore, M., Jackman, J., Lesczynska, D., \& Leszczynski, J. (2012). Structures of gold clusters in the size range 2-2016 atoms. A quantum-chemical study by Extended Huckel and DFT. Nanoscience \& Nanotechnology-Asia, 2(1), 2-10.

Smanova, Z. A., Gafurova, D. A., \& Savchkov, A. V. (2011). Disodium 1-(2-Pyridylazo)-2-oxynaphthalene3,6-disulfonate: An Immobilized Reagent for Iron(III) Determination. Russian Journal of Organic Chemistry, 81(4), 739-742. doi:10.1134/S1070363211040207

Wang, H., \& Lu, J. (2020). A review on particle size effect in metal-catalyzed heterogeneous reactions. Chinese Journal of Chemistry, 38(11), 1422-1444. doi:10.1002/cjoc.202000205 